\title{
What do we know about DNA mechanics so far? Abhishek Aggarwal ${ }^{1,4}$, Supriyo Naskar ${ }^{1,4}$, Anil Kumar Sahoo ${ }^{1,4}$, Santosh Mogurampelly ${ }^{2}$, Ashok Garai ${ }^{3}$ and Prabal K Maiti ${ }^{1}$
}

The DNA molecule, apart from carrying the genetic information, plays a crucial role in a variety of biological processes and finds applications in drug design, nanotechnology and

nanoelectronics. The molecule undergoes significant structural transitions under the influence of forces due to physiological and non-physiological environments. Here, we summarize the insights gained from simulations and single-molecule experiments on the structural transitions and mechanics of DNA under force, as well as its elastic properties, in various environmental conditions, and discuss appealing future directions.

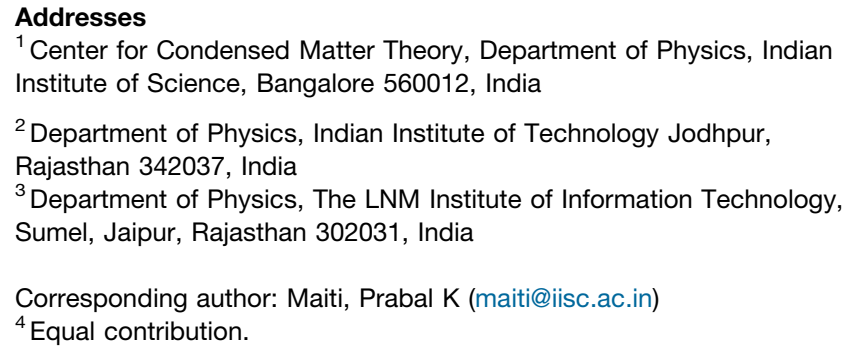

${ }^{3}$ Department of Physics, The LNM Institute of Information Technology, Sumel, Jaipur, Rajasthan 302031, India

Corresponding author: Maiti, Prabal K (maiti@iisc.ac.in)

${ }^{4}$ Equal contribution.

\author{
Current Opinion in Structural Biology 2020, 64:42-50 \\ This review comes from a themed issue on Biophysical and com- \\ putational methods \\ Edited by Nagasuma Chandra and Gautam I Menon \\ For a complete overview see the Issue and the Editorial \\ Available online 29th June 2020 \\ https://doi.org/10.1016/j.sbi.2020.05.010 \\ 0959-440X/@ 2020 Elsevier Ltd. All rights reserved.
}

\section{Introduction}

The celebrated molecule of life, DNA is one of the most intensively studied biopolymers because of its central role in molecular and cell biology. DNA molecules are often bent, twisted, and stretched, as well as undergo various structural transitions, due to the influence of different forces arising from its biological or artificial environments [1]. DNA structural transitions play a crucial role in facilitating numerous biological functions. A deeper insight into the nature of DNA structural transitions under mechanical forces offer an excellent route to characterize the mechanical and elastic properties of DNA. Moreover, the understanding of the mechanisms of the underlying DNA structural transitions has important implications for DNA-based nano-technological applications, such as rational drug design, programmable molecular machines, and DNA origami. Due to the advent of single-molecule manipulation techniques and theoretical/computational studies, significant progress has been made in understanding the structural, mechanical and elastic properties of DNA [1-12], as well as in understanding small-molecule interactions with DNA $\left[13,14,15^{\circ}\right]$.

In this minireview, we focus on some of the recent key developments in the field of DNA mechanics. Different theoretical models of DNA elasticity are first discussed, followed by discussions on the changes in DNA elasticity in various environmental conditions, as well as DNA structural transitions, force-extension behavior, and strand separation (or melting) under external force.

\section{Theoretical models to study DNA mechanics}

Double-stranded DNA (dsDNA) undergoes various deformations namely twisting, bending and stretching during various transcription and genomic processes [16]. Numerous models have been proposed to understand and quantify the DNA mechanical properties $\left[6,17^{\bullet \bullet}, 18,19,20,21^{\bullet \bullet}, 22,23,66\right]$. The most widely used model is the Worm-like Chain (WLC) model which characterizes dsDNA as an elastic rod that bends smoothly under the random thermal fluctuations (Figure 1a). This model consists of a single fitting parameter termed as 'persistence length' and assumes quadratic form of bending deformation energies and is used extensively to calculate various elastic properties of dsDNA. The WLC model corresponds to the isotropic elastic rod model in the limit of no twist and no stretch [23]. Within the framework of elastic rod model, the energy $(E)$ of a nucleic acid (NA) under the application of an external force $(F)$ can be written as [24]

$$
\begin{aligned}
E= & E_{\text {Stretch }}+E_{\text {Twist }}+E_{T \text { wist-stretch }}+E_{\text {Bend }} \\
- \text { force } & \times \text { extension } \\
\Rightarrow E & =\frac{1}{2} \frac{\gamma}{L}(\delta L)^{2}+\frac{1}{2} \frac{C}{L} \varphi^{2}+\frac{g}{L}(\delta L) \cdot \varphi \\
& +\frac{1}{2} \frac{\kappa}{L} \theta^{2}-F \cdot(\delta L)
\end{aligned}
$$

where $L$ is the equilibrium contour length, $\delta L$ is the extension in $L$, and $\varphi$ and $\theta$ are the change in helical twist and bending angle, respectively from their equilibrium values. The symbols $\gamma, C, g$ and $\kappa$ are the stretch 


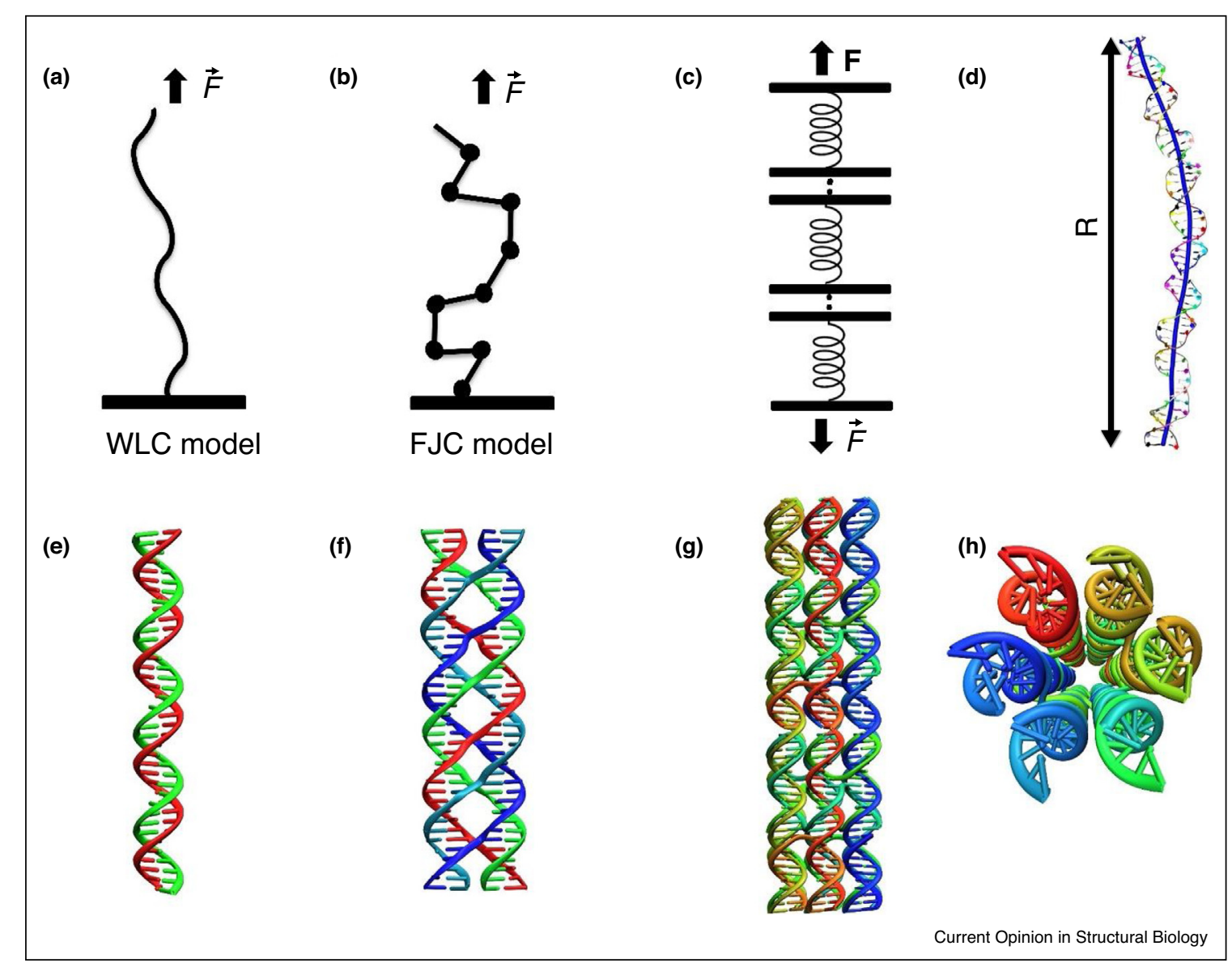

Schematic diagrams of different models to measure dsDNA and DNA nanostructure's elasticity. (a) Freely Jointed Chain (FJC) model, (b) Wormlike Chain (WLC) model. The external force is represented by the black arrow. (c) Schematic diagram to represent the dsDNA base pairs connected via springs. (d) Schematic diagram to show the flexibility of long dsDNA chain. The blue line represents the central axis of dsDNA, whereas $\mathrm{R}$ represents the end-to-end length of the dsDNA. Clearly, the contour length of the dsDNA is very different than the sum of distances between consecutive base pairs. (e-h) Computational models of dsDNA and DNA based nanostructures where these models have been implemented. (e) 38 bp dsDNA, (f) Paranemic crossover DNA molecule. (g) Side view and (h) top view of DNA nanotube.

modulus, twist modulus, twist-stretch coupling constant and bending modulus, respectively. In the absence of any external force, the Boltzmann probability distribution of contour length and bending angle can be written as $\left[15^{\circ \bullet}\right.$ $\left., 17^{\bullet \bullet}, 25,26^{\bullet}, 27,28\right]$

$P(L)=\sqrt{\frac{\gamma}{2 \pi K_{B} T L}} \exp \left[-\frac{\gamma}{2 K_{B} T L}(\delta L)^{2}\right]$

$P(\theta)=\sqrt{\frac{\kappa}{2 \pi K_{B} T L}} \exp \left[-\frac{\kappa}{2 K_{B} T L} \theta^{2}\right]$

Many recent studies have implemented the above relations to compute $\gamma, \kappa$ and persistence length $\left(L_{p}=\frac{\kappa}{K_{B} T}\right)$ of nucleic acids $\left[15^{\bullet \bullet}, 17^{\bullet \bullet}, 25,26^{\bullet}\right]$. Mean square end-to-end distance $(R)$ of the worm-like chain is given by [29]

$R^{2}=2 L_{p} L_{0}-2 L_{p}^{2}\left\{1-\exp \left(-\frac{L_{0}}{L_{p}}\right)\right\}$

where $L_{O}$ is the contour length of the chain. This equation is a smooth crossover between an ideal chain $\left(L_{0}>>L_{p}\right)$ and a rod-like chain $\left(L_{0}<<L_{p}\right)$. Thus, the above Eq. becomes very useful to measure the persistence length of DNA [17 ${ }^{\circ}$ ]. Maaloum et al. studied the flexibility of short DNA using AFM experiments and found that the flexibility of short DNA, beyond two helical turns, can be well captured by the WLC model [28,30]. Maiti and co-workers implemented the WLC model to study the mechanics of dsDNA and DNA based nanostructures like paranemic crossover DNA molecule, DNA nanotube, and so on, and 
found their elastic properties to be in close agreement with the experimental results (Figure 1) [31,32,33 $\left.{ }^{\bullet}, 34\right]$. Golestanian et al. found that the stretch, bend and twist of the DNA depend on its length. Their study indicates that shorter DNAs are more flexible than the longer ones [35]. They believe that this softening is associated with the 'cooperative' motions of the DNA base pairs rather than its completely independent random motions. The validity of WLC model for short dsDNA molecules and at high force regimes is however challenged by some experimental studies [36]. Several modifications to WLC model $[11,12,22,23]$ have been proposed to explain the formation of bubbles and kinks in dsDNA. The limit of the applicability of WLC model for certain length of dsDNA remains debatable $\left[17^{\bullet \bullet}, 37\right]$. Gross et al. [22] presented a twistable WLC (tWLC) model where an enthalpic twiststretch coupling term is added to the WLC model which accounts for the changes in the DNA elongation due to variations in the twist. This description includes both the helicity and the sequence of the dsDNA and accurately explains the experimentally observed force-extension curves at all force regimes. In tWLC model, the relation between $F$ and $\delta L$ is given by [38]:

$\delta \mathrm{L}=L\left(1-\frac{1}{2} \sqrt{\frac{K_{B} T}{F \cdot L_{p}}}\right)+\frac{C}{-g^{2}+\gamma C}$

For WLC model, that is, in the limit of $C \rightarrow 0$, Eq. (6) reduces to $\delta \mathrm{L}=L\left(1-\frac{1}{2} \sqrt{\frac{K_{B} T}{F \cdot L_{p}}}\right)$ and the $L_{p}$ can be obtained from the slope of the curve between $\delta L$ and $F$.

Linear Sub-Elastic Chain (LSEG) model [19] is also proposed to quantify the DNA elastic properties which considers linear energy function for the bending energy of dsDNA as opposed to the second order energy function in WLC model. Other than WLC model, Freely Jointed Chain (FJC) model [6] is also used to describe the dsDNA mechanics. FJC model assumes a polymer chain consisting of $n$ segments of characteristic length (Kuhn length), connected via freely rotating joints [6,23] (Figure 1b). However, FJC model can only describe the behavior of dsDNA in the limit of low forces but fails to do so at intermediate and higher forces [6]. Lipfert et al. [20,39] proposed springiness model to explain the opposite twist-stretch coupling of dsRNA relative to dsDNA where dsRNA's 'spring-like' properties are expected to render it more pliable to stretching. Inspired by the springiness model, Perez et al. [21 $\left.{ }^{\bullet \bullet}\right]$ recently suggested a new parameter termed as 'Crookedness' parameter to regulate the DNA mechanics at short length scales which is defined as the cosine inverse of the ratio of end-to-end length of the dsDNA to its contour length (Figure 1d). To link DNA crookedness to its stretch modulus, DNA base pairs are assumed to be beads connected via springs (Figure 1c). A low crookedness is found to be related to the high DNA stretching stiffness in their work. This model predicts the sequence-dependent elastic properties of dsDNA quite well.

\section{DNA in different environments}

The diverse intracellular physiological conditions surrounding DNA, in terms of ionic atmosphere, neighboring proteins and biomolecules, have a strong influence on its mechanical properties (Figure 2). Being a highly negatively charged biomolecule, the folding and compaction of DNA require overcoming an enormous electrostatic energy barrier, which is screened by the presence of positive counterions, proteins, and other biomolecules. It is intriguing how a relatively stiff and charged DNA molecule wraps around a histone octamer or forms DNA-protein complexes [40]. The mechanical properties of DNA thus change with its environment to facilitate it to make stable complexes. Of all such factors, ions influence the DNA mechanics the most in various processes like transcription, replication, DNA repair, DNA packing, and chromosome formation [41]. Recent theoretical and experimental studies reveal that with increasing cation concentration, DNA bending flexibility increases and thus its persistence length decreases. Recently, Garai et al. observed the opposing effect of increasing monovalent salt concentration on the bending flexibility and the stretch modulus of short dsDNA [17 $\left.{ }^{\bullet \bullet}\right]$. Increasing salt concentration enhances the screening of DNA backbone charges, which makes dsDNA easier to bend but harder to stretch. Drozdetski et al. showed that multivalent ions decrease the bending rigidity of B-form dsDNA, whereas A-form dsRNA bending rigidity increases by $30 \%$ [42]. In contrast, Broekmans $e t a l$. found from the force-extension data that the dsDNA persistence length is insensitive to the variation in magnesium concentration [43]. Though the DNA-ion interaction is well understood, it is extremely challenging to theoretically model and quantitively explain the effect of ion interaction on DNA mechanical properties. Guilbaud et al. combining Netz-Orland [44] and Trizac-Shen [45] formalism with nonlinear electrostatic effect on a charged polymer, quantitatively describe their experimental data of dsDNA persistence length over a large range of ionic concentration for various metal ions, such as $\mathrm{Na}^{+}, \mathrm{K}^{+}, \mathrm{Li}^{+}, \mathrm{Mg}^{2+}$ and $\mathrm{Ca}^{2+}$ $\left[46^{\bullet \bullet}\right.$. Several other studies on DNA-ion interaction reinforce the above observations $[47,48]$. In contrast to the behavior exhibited by dsDNA in salt solution, both dsDNA persistence length and stretch modulus increase with ionic liquid (IL) concentration [26 $6^{\circ}$. IL cations infiltrate into the grooves of dsDNA where they establish strong electrostatic and hydrophobic interactions with the nucleobases and the sugar moieties and subsequently increase dsDNA rigidity. When dsDNA comes in the vicinity of macromolecules like proteins, dendrimers, intercalators (Figure 2), the DNA mechanics changes drastically. Binding of proteins on DNA significantly changes its elastic response [49]. Positively charged dendrimer decreases the dsDNA persistence length by neutralizing the backbone charges, 


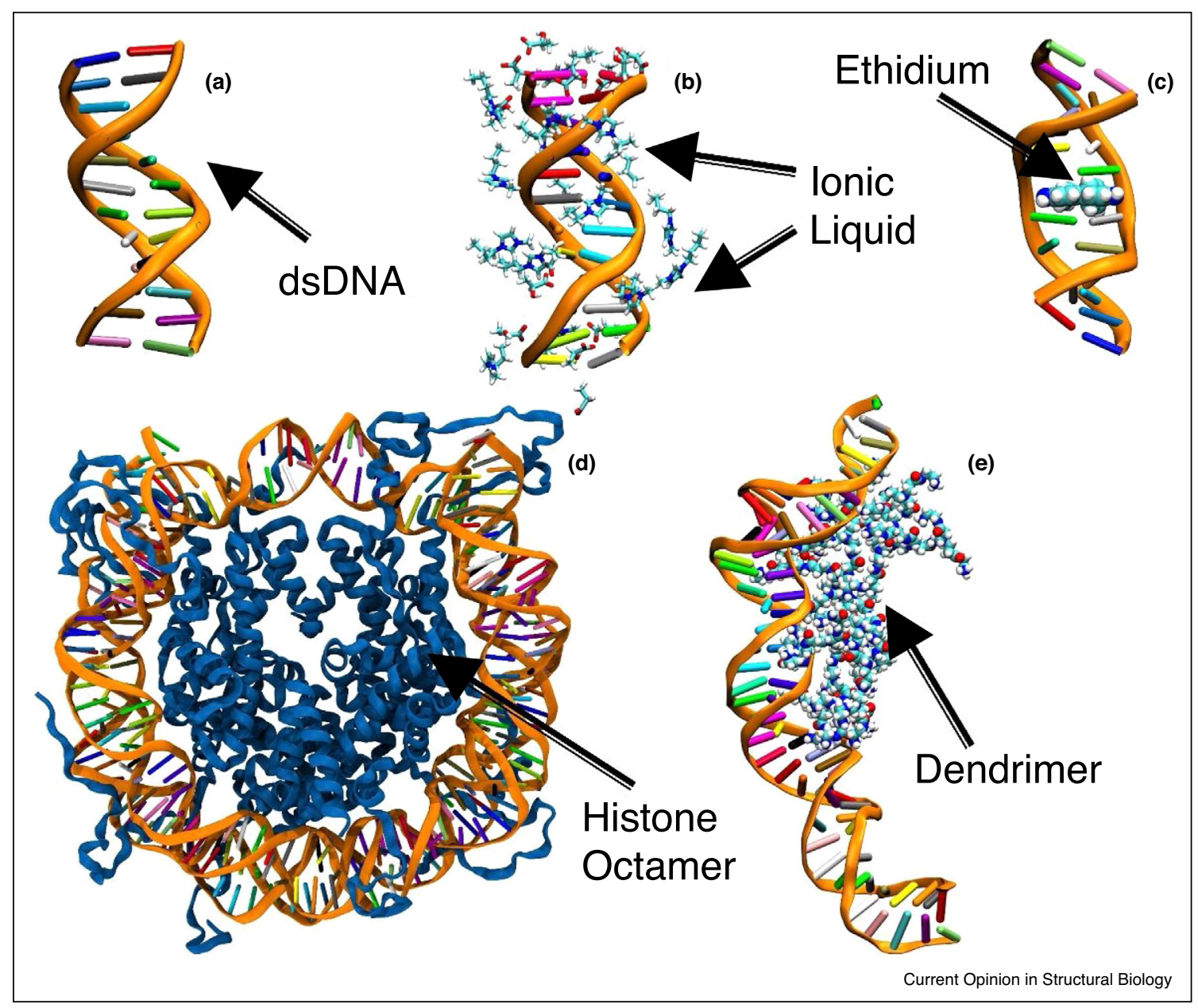

Equilibrated atomistic structures of dsDNA in various environments. Molecular structures of MD simulated (a) dsDNA in aqueous conditions, (b) dsDNA in ionic liquid solution, (c) dsDNA intercalated with ethidium molecule, (d) nucleosome core particle (NCP), and (e) DNA-dendrimer complex. Water molecules are not shown here for clarity.

whereas the stretch modulus remains unaffected [25]. In contrast, once dsDNA makes a complex with the positively charged histone octamer that partially neutralizes DNA backbone charges, both its bending and stretch modulus increase by almost four times $\left[17^{\circ \bullet}\right]$. Sahoo et al. recently discovered that when drug molecules bind to dsDNA in the intercalation mode, the stretch modulus increases drastically, whereas the persistence length decreases similar to the scenario of dsDNA in monovalent salt solutions [ $\left[15^{\circ \bullet}\right]$. However, there exist conflicting reports in the literature claiming no change in DNA persistence length upon drug intercalation, as discussed in Refs. $\left[15^{\bullet \bullet}, 50\right]$. The mechanical properties of dsDNA in various environments are summarized in Table 1.

\section{Structural transitions in dsDNA under force}

dsDNA exhibits different force-extension regimes with Hookean behavior at low forces and a drastic elongation of its length at a force of $65 \mathrm{pN}[1,6]$. This is followed by an overstretching behavior where dsDNA transforms to a completely new structural phase-broadly described as a thermodynamically melted form (with broken WatsonCrick base-pairs) or a structurally intact base-pair stacking alignment in a ladder-like fashion (the so-called S-DNA) [6,51-53].

In the force induced melting perspective, Bloomfield et al. [8] proposed a theoretical model based on the underlying thermodynamics to explain quantitatively the experimentally observed overstretching transition. Dynamics of DNA under external force in a coarse grain level can be found in a review by Kumar and Li [54]. Computer simulations using atomistic models by Maiti and co-workers $\left[4,5,15^{\bullet}, 33^{\bullet}, 51,52,55\right]$ expanded the understanding of the nature of underlying mechanisms with microscopic details and provided deeper insights 


\begin{tabular}{|c|c|c|c|}
\hline \multicolumn{4}{|c|}{ Mechanical properties of dsDNA in various environment } \\
\hline System & Persistence length $(\mathrm{nm})$ & Stretch modulus $(\mathrm{pN})$ & Reference \\
\hline dsDNA & $49.89 \pm 0.77$ & $\begin{array}{l}1096 \text { to } \\
1280\end{array}$ & {$\left[17^{\bullet \bullet}, 24\right]$} \\
\hline dsDNA in $250 \mathrm{mM} \mathrm{NaCl}$ & $33.16 \pm 0.45$ & $2345 \pm 65$ & {$\left[17^{\bullet \bullet}\right]$} \\
\hline NCP DNA & $59.02 \pm 6.24$ & $4021 \pm 151$ & {$\left[17^{\circ \bullet}\right]$} \\
\hline ethidium intercalated dsDNA & $30.97 \pm 1.77$ & $2118.6 \pm 102.3$ & {$\left[15^{\circ}\right]$} \\
\hline $\begin{array}{l}\text { dsDNA-dendrimer } \\
\text { complex }\end{array}$ & 6.3 & 959 & [25] \\
\hline dsDNA in 1-butyl-3-methyl imidazolium acetate & $248.74 \pm 6.8$ & $3926.24 \pm 124.02$ & {$\left[26^{\circ}\right]$} \\
\hline Bare dsDNA in Physiological Condition (experiment) & 39 to 50 & 1450 to 1750 & {$[38,43]$} \\
\hline
\end{tabular}

into the force induced DNA melting description of the DNA structural transitions. Besides, several researchers reported the observation of S-DNA, under specific force attachments at the end points of DNA strands by using both single-molecule experiments and computer simulations $[5,7,9,10]$ (Figure 3).

While the nature of DNA structural phase transitions under applied forces has been well understood from a broad perspective, there are few challenges in expanding the application spectrum of DNA-based materials $\left[32,33^{\circ}, 56\right]$. Firstly, there is a lack of fundamental understanding of the dynamics of DNA undergoing overstretching transition due to interaction with proteins and other biomolecules, the orientation of base-pair tilt and its sensitivity with respect to the presence of biomacromolecules and so on. Despite the requirement for a thorough examination of the above subject in the context of DNA role in rational drug design applications, there were only a few preliminary reports dealing with the above-mentioned issues. Mameren et al. [57] investigated the molecular level dynamic features exhibited by intercalated dsDNA under a large range of pulling forces using polarized fluorescence microscopy in conjunction with optical-tweezers-based single-molecule manipulation techniques (Figure 3a). They measured the alignment of the transition dipoles of the intercalating dyes when the DNA is pulled tightly. On average, the intercalated dyes are oriented perpendicular to the long axis of the DNA. Moreover, the dyes undergo fast dynamics on the time scales of absorption and fluorescence emission processes. It is also reported that the intercalating dyes remain bound to DNA even beyond overstretching forces where DNA is melted. Peterman et al. [58] used single-molecule polarization microscopy to examine the structure of S-DNA with the help of intercalated dyes in the regime of DNA under tension. In contrast to the perpendicular orientations typically observed at low stretching forces, they find that the intercalator dyes adopt a tilted orientation of $54^{\circ}$ relative to the DNA long axis at stretching forces beyond the dramatic overstretching transition. It has been concluded that the S-DNA has substantially inclined base pairs relative to those of the canonical
B-DNA conformation. Note that the novel approach devised by Peterman et al. [58] is a significant achievement in determining the inclination of intercalated dye molecules. However, it is not possible to directly measure the orientations of the DNA base-pairs in overstretched regime and therefore it is hard to examine the effect of intercalators on the base-pair inclination of overstretched form of DNA (specifically in this context S-DNA). Further efforts are required to establish a complete picture of structural basis for the DNA conformational changes that enable genomic transactions and biotechnological processing of DNA. In this context, the drug design applications based on DNA require more serious efforts.

\section{Force-induced DNA strand separation}

In various biological processes, such as DNA transcription (with the help of RNA polymerases), replication (with the help of helicases), and repair, the two strands of a dsDNA are melted from the middle or ends of the DNA, either partially or fully [1]. The design principle of a variety of drug molecules, including that for cancer and tumour treatments, is based on the principle that these molecules bind to DNA and make dsDNA melting extremely difficult, which thus could hamper the biological processes required for proper functioning of the cellular processes. In this context, Sahoo et al. $\left[15^{\circ \bullet}\right]$ employed steered molecular dynamics (MD) simulations of different pulling protocols to study the overstretching and melting transitions of dsDNA and found a significant increase in the dsDNA rupture force in the presence of intercalated drug molecules, which is due to the enhancement in the base-stacking interaction between adjacent base pairs present near to a drug intercalation site (Figure 3). This is in line with the recent revelation that the base-stacking interactions, instead of the previously thought base-pairing interactions, mostly contribute to the stability of dsDNA [59]. Moreover, they found that in the presence of intercalated drug molecules, dsDNA stretch modulus increases significantly, in agreement with several experimental reports $\left[60^{\circ}, 61\right]$. Their study revealed that anticancer drugs could function by altering the mechanical properties of DNA. 


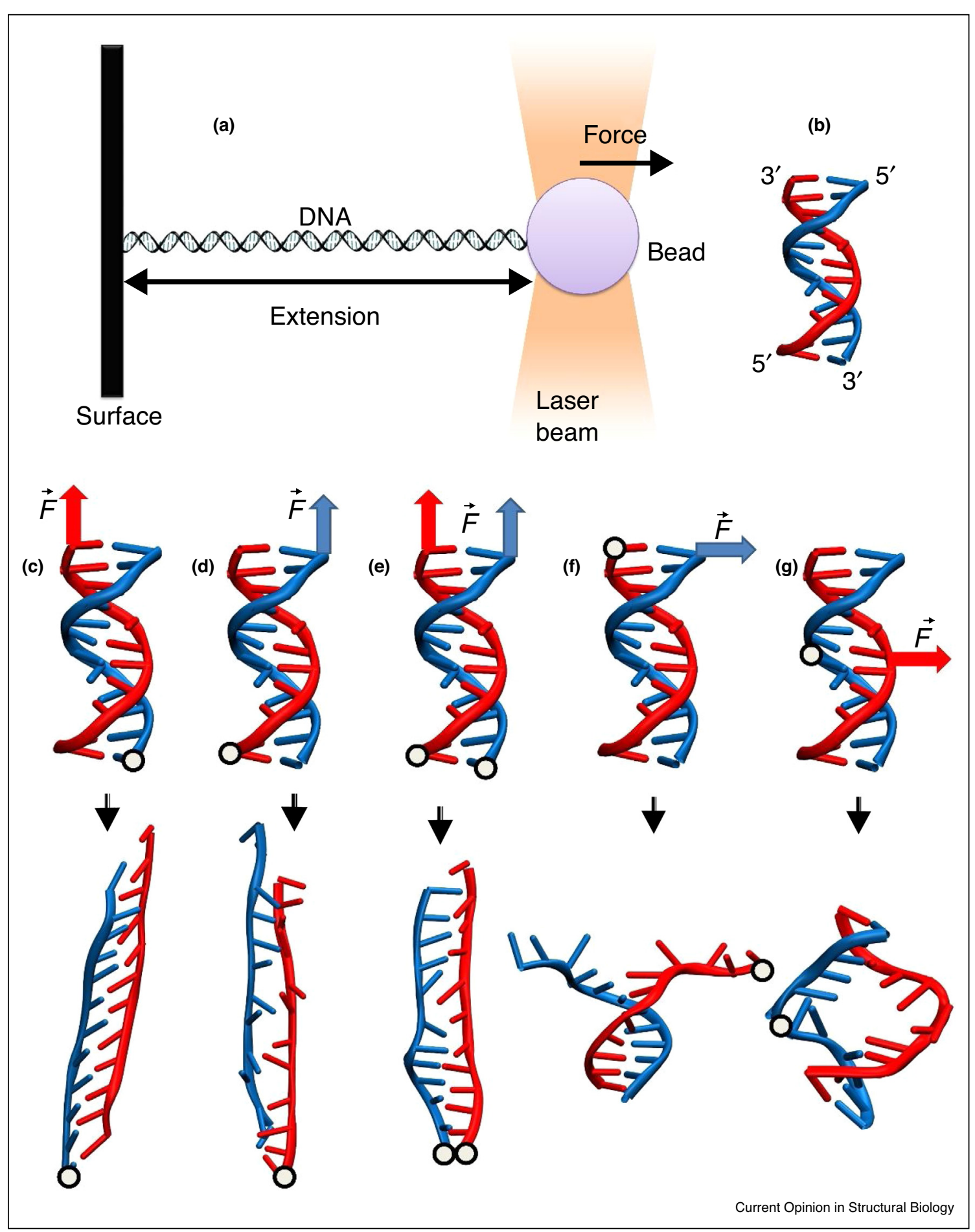

Pulling protocol dependent structural transitions of dsDNA. (a) Schematic diagram to represent the experimental setup to stretch dsDNA using optical tweezers. (b) Schematic diagram to highlight the 3' and 5' ends of dsDNA. (c-g) Stretching dsDNA using various pulling protocols. Here, the black circle represents the fixed end of dsDNA while red and blue arrows represent the direction of force applied to dsDNA along (c) 3' end, (d) 5' end, (e) both 3' and 5' ends in the same side, (f) unzipping force applied at terminal base of dsDNA and (g) unzipping force applied at the middle of dsDNA. Stretching dsDNA along 3' end leads to a ladder-like S-form of DNA, whereas stretching along 5' end leads to melting of dsDNA. Stretching a dsDNA along both 3' and 5' ends leads to an intermediate state where some portions of DNA show ordered base pairs, while others show melting. Unzipping dsDNA along ends and middle of dsDNA leads to its tearing in different fashions. 
DNA methylation (or hydroxymethylation) is known to play a vital role in epigenetic regulation of gene expression. A combined experimental and simulation study [62] revealed that the rupture force for DNA strand separation increases upon methylation of its Cytosine bases due to the enhancement in base-stacking interaction. They also showed that the rupture force not only depends on the methylation level but also on the sequence of methylated bases. The same but more pronounced effects were observed for Cytosine hydroxymethylation [55]. These studies thus provided a mechanism that methylation or hydroxymethylation, via altering DNA mechanical properties, could control biological functions.

Many DNA-associated biological processes hinge on sequence-selective molecular interactions [63]. By using the force-induced remnant magnetization spectroscopy technique, $\mathrm{Hu}$ et al. [63] measured the differential binding force to demonstrate the DNA sequence-specific binding of daunomycin (an anticancer drug), as well as to reveal the enantioselective binding of tetrahydropalmatine (L or D form) with different DNA sequencesthe understanding of which has important implications for pharmaceutical research. Manosas et al. [64] used an assay of mechanical unzipping of DNA hairpins to determine sequence-selective binding of small ligands. This study revealed that actinomycin (a mono-intercalator) likes to bind $\mathrm{GpC}$ steps, whereas bis-intercalators thiocoraline and echinomycin like to bind $\mathrm{CpG}$ steps.

\section{Conclusions and future perspectives}

Advances in single-molecule experiment techniques and insights from computational simulations have greatly expanded our understanding of DNA mechanics, DNA-associated cellular processes, and functioning of various drug molecules that target DNA. dsDNA has been studied in various conditions, such as unzipping, shearing, stretching, and under a torque, to mimic different biological scenarios [1]. These have provided increased insights to various interesting aspects of DNA such as emergence of S-DNA upon overstretching [5,10,51,52], modifications of DNA structural and mechanical properties under different environments such as drug intercalators $\left[15^{\bullet \bullet}\right]$, salts $\left[17^{\bullet \bullet}, 46^{\bullet \bullet}\right]$ and ionic liquids $\left[26^{\circ}\right]$. These information are the key inputs for DNA nanotechnological applications, enabling researchers to design various DNA nanostructures like DNA nanotubes $\left[32,33^{\circ}\right]$ and DNA nanorobots for drug delivery [65], as well as in DNA nanoelectronics [51,52].

Use of DNA for nanobiotechnological applications still requires a complete understanding of its mechanical properties at different length scales and in complex and crowded environments. Further investigations, combining experiment, simulation, and theory, are required to fully understand the dependence of DNA persistence length on its contour length and sequence, as well as its interaction with salts, proteins, and small-molecule ligands. How confinement affects DNA structural and elastic properties will be an important direction to pursue from theoretical/simulation point of view, as it has high relevance for cell biology, it is less studied and understood, and probing it experimentally is a rather difficult task. Moreover, the structural transitions of DNA in confined geometry, under the application of an external force can be obtained with unprecedented resolution from molecular simulations.

\section{Conflict of interest statement}

Nothing declared.

\section{Acknowledgements}

A.A., S.N., and A.K.S. thank MHRD India, CSIR India, and IISc, respectively for the generous fellowship. We thank DAE, India for financial support. AG thanks SERB, DST, Govt. of India for the financial support (grant no ECR/2017/000683).

\section{References and recommended reading}

Papers of particular interest, published within the period of review, have been highlighted as:

- of special interest

-• of outstanding interest

1. Bustamante $C$, Bryant $Z$, Smith SB: Ten years of tension: singlemolecule DNA mechanics. Nature 2003, 421:423-427.

2. Bustamante C, Marko J, Siggia E, Smith S: Entropic elasticity of \$ Vlambda \$-phage DNA. Proc Natl Acad Sci U S A 1991, 88:10009.

3. Marko JF, Siggia ED: Stretching DNA. Macromolecules 1995, 28:8759-8770.

4. Santosh M, Maiti PK: Force induced DNA melting. J Phys: Condensed Matter 2008, 21034113.

5. Garai A, Mogurampelly S, Bag S, Maiti PK: Overstretching of BDNA with various pulling protocols: appearance of structural polymorphism and S-DNA. J Chem Phys 2017, 147:225102.

6. Smith SB, Cui Y, Bustamante C: Overstretching B-DNA: the elastic response of individual double-stranded and singlestranded DNA molecules. Science 1996, 271:795-799.

7. Lebrun A, Lavery R: Modelling extreme stretching of DNA. Nucleic Acids Res 1996, 24:2260-2267.

8. Rouzina I, Bloomfield VA: Force-induced melting of the DNA double helix 1. Thermodynamic analysis. Biophys J 2001, 80:882-893.

9. van Mameren J, Gross P, Farge G, Hooijman P, Modesti M, Falkenberg M, Wuite GJ, Peterman EJ: Unraveling the structure of DNA during overstretching by using multicolor, singlemolecule fluorescence imaging. Proc Natl Acad Sci U S A 2009, 106:18231-18236

10. Danilowicz C, Limouse C, Hatch K, Conover A, Coljee VW, Kleckner N, Prentiss M: The structure of DNA overstretched from the $5^{\prime} 5^{\prime}$ ends differs from the structure of DNA overstretched from the $3^{\prime} 3^{\prime}$ ends. Proc Natl Acad Sci U S A 2009, 106:13196-13201.

11. Ranjith P, Kumar PS, Menon GI: Distribution functions, loop formation probabilities, and force-extension relations in a model for short double-stranded DNA molecules. Phys Rev Lett 2005, 94:138102.

12. Ranjith P, Menon GI: Stretching and bending fluctuations of short DNA molecules. Biophys J 2013, 104:463-471. 
13. Almaqwashi AA, Paramanathan T, Rouzina I, Williams MC: Mechanisms of small molecule-DNA interactions probed by single-molecule force spectroscopy. Nucleic Acids Res 2016, 44:3971-3988.

14. Chaurasiya KR, Paramanathan T, McCauley MJ, Williams MC: Biophysical characterization of DNA binding from single molecule force measurements. Phys Life Rev 2010, 7:299-341.

15. Sahoo AK, Bagchi B, Maiti PK: Understanding enhanced

-. mechanical stability of DNA in the presence of intercalated anticancer drug: implications for DNA associated processes J Chem Phys 2019, 151:164902

Extensive all-atom simulation study probing the changes in the structure, elasticity, and strand-dissociation mechanics of DNA in the presence of anticancer drugs.

16. Blackwood EM, Kadonaga JT: Going the distance: a current view of enhancer action. Science 1998, 281:60-63.

17. Garai A, Saurabh S, Lansac Y, Maiti PK: DNA elasticity from

- $\quad$ short DNA to nucleosomal DNA. J Phys Chem B 2015 , 119:11146-11156

First extensive all-atom simulation study to calculate the elastic properties of nucleosome DNA and comparing various theories to compare the DNA elastic properties.

18. Kratky O, Porod G: Röntgenuntersuchung gelöster fadenmoleküle. Recueil des Travaux Chimiques des Pays-Bas 1949, 68:1106-1122.

19. Wiggins PA, Van Der Heijden T, Moreno-Herrero F, Spakowitz A Phillips R, Widom J, Dekker C, Nelson PC: High flexibility of DNA on short length scales probed by atomic force microscopy. Nat Nanotechnol 2006, 1:137.

20. Chou F-C, Lipfert J, Das R: Blind predictions of DNA and RNA tweezers experiments with force and torque. PLOS Comput Biol 2014, 10:e1003756.

21. Marin-Gonzalez A, Vilhena J, Moreno-Herrero F, Perez R: DNA

-• crookedness regulates DNA mechanical properties at short length scales. Phys Rev Lett 2019, 122048102

Provides a new parameter to quantify the DNA flexibility and bending.

22. Gross $P$, Laurens N, Oddershede LB, Bockelmann U, Peterman EJ, Wuite GJ: Quantifying how DNA stretches, melts and changes twist under tension. Nat Phys 2011, 7:731-736.

23. Camunas-Soler J, Ribezzi-Crivellari M, Ritort F: Elastic properties of nucleic acids by single-molecule force spectroscopy. Annu Rev Biophys 2016, 45:65-84.

24. Marin-Gonzalez A, Vilhena JG, Perez R, Moreno-Herrero F: Understanding the mechanical response of double-stranded DNA and RNA under constant stretching forces using all-atom molecular dynamics. Proc Natl Acad Sci U S A 2017, 114:70497054 .

25. Mogurampelly S, Nandy B, Netz RR, Maiti PK: Elasticity of DNA and the effect of dendrimer binding. Eur Phys J E 2013, 36:68.

26. Garai A, Ghoshdastidar D, Senapati S, Maiti PK: lonic liquids

- $\quad$ make DNA rigid. J Chem Phys 2018, 149045104

The first all-atom MD simulation study of DNA elastic properties in ionic liquids.

27. Mazur AK: Wormlike chain theory and bending of short DNA Phys Rev Lett 2007, 98:218102.

28. Mazur AK, Maaloum M: DNA flexibility on short length scales probed by atomic force microscopy. Phys Rev Lett 2014, 112 068104.

29. Rubinstein M, Colby RH: Polymer Physics. New York: Oxford University Press; 2003.

30. Mazur AK, Maaloum M: Atomic force microscopy study of DNA flexibility on short length scales: smooth bending versus kinking. Nucleic Acids Res 2014, 42:14006-14012.

31. Santosh M, Maiti PK: Structural rigidity of paranemic crossover and juxtapose DNA nanostructures. Biophys J 2011, 101:1393-1402.

32. Naskar S, Gosika M, Joshi H, Maiti PK: Tuning the stability of DNA nanotubes with salt. J Phys Chem C 2019, 123:9461-9470.
33. Joshi H, Kaushik A, Seeman NC, Maiti PK: Nanoscale structure

- $\quad$ and elasticity of pillared DNA nanotubes. ACS Nano 2016, 10:7780-7791

First all-atom simulation study reporting the mechanical properties of DNA nanostructures.

34. Naskar S, Joshi H, Chakraborty B, Seeman NC, Maiti PK: Atomic structures of RNA nanotubes and their comparison with DNA nanotubes. Nanoscale 2019, 11:14863-14878.

35. Noy A, Golestanian R: Length scale dependence of DNA mechanical properties. Phys Rev Lett 2012, 109:228101.

36. Yuan C, Chen H, Lou XW, Archer LA: DNA bending stiffness on small length scales. Phys Rev Lett 2008, 100:018102.

37. Xiao S, Liang $\mathrm{H}$, Wales DJ: The contribution of backbone electrostatic repulsion to DNA mechanical properties is length-scale-dependent. J Phys Chem Lett 2019, 10:4829-4835.

38. Gross $P$, Laurens $N$, Oddershede LB, Bockelmann U, Peterman EJG, Wuite GJL: Quantifying how DNA stretches, melts and changes twist under tension. Nat Phys 2011 7:731-736.

39. Liebl K, Drsata T, Lankas F, Lipfert J, Zacharias M: Explaining the striking difference in twist-stretch coupling between DNA and RNA: a comparative molecular dynamics analysis. Nucleic Acids Res 2015, 43:10143-10156.

40. Richmond TJ, Davey CA: The structure of DNA in the nucleosome core. Nature 2003, 423:145.

41. Baumann CG, Smith SB, Bloomfield VA, Bustamante C: Ionic effects on the elasticity of single DNA molecules. Proc Natl Acad Sci U S A 1997, 94:6185-6190.

42. Drozdetski AV, Tolokh IS, Pollack L, Baker N, Onufriev AV: Opposing effects of multivalent ions on the flexibility of DNA and RNA. Phys Rev Lett 2016, 117:028101.

43. Broekmans OD, King GA, Stephens GJ, Wuite GJ: DNA twist stability changes with magnesium $(2+)$ concentration. Phys Rev Lett 2016, 116:258102.

44. Netz RR, Orland $\mathrm{H}$ : Variational charge renormalization in charged systems. Eur Phys J E 2003, 11:301-311.

45. Trizac E, Shen T: Bending stiff charged polymers: the electrostatic persistence length. EPL (Europhysics Letters) 2016, 116:18007.

46. Guilbaud S, Salomé L, Destainville N, Manghi M, Tardin C:

-• Dependence of DNA persistence length on ionic strength and ion type. Phys Rev Lett 2019, 122:028102

Combined experiment and theoretical study to measure DNA persistence length in different salts.

47. Sun T, Mirzoev A, Minhas V, Korolev N, Lyubartsev AP, Nordenskiöld L: A multiscale analysis of DNA phase separation: from atomistic to mesoscale level. Nucleic Acids Res 2019 , 47:5550-5562.

48. Brunet A, Tardin C, Salome L, Rousseau P, Destainville N, Manghi M: Dependence of DNA persistence length on ionic strength of solutions with monovalent and divalent salts: a joint theory-experiment study. Macromolecules 2015, 48:3641-3652

49. Yan J, Marko JF: Effects of DNA-distorting proteins on DNA elastic response. Phys Rev E 2003, 68:011905.

50. Rocha M: Extracting physical chemistry from mechanics: a new approach to investigate DNA interactions with drugs and proteins in single molecule experiments. Integr Biol 2015, 7:967-986.

51. Bag S, Mogurampelly S, Goddard WA III, Maiti PK: Dramatic changes in DNA conductance with stretching: structural polymorphism at a critical extension. Nanoscale 2016, 8:1604416052.

52. Aggarwal A, Bag S, Maiti PK: Remarkable similarity of force induced dsRNA conformational changes to stretched dsDNA and their detection using electrical measurements. Phys Chem Chem Phys 2018, 20:28920-28928. 
53. Cluzel P, Lebrun A, Heller C, Lavery R, Viovy J-L, Chatenay D, Caron F: DNA: an extensible molecule. Science 1996, 271:792-794.

54. Kumar S, Li MS: Biomolecules under mechanical force. Phys Rep 2010, 486:1-74.

55. Severin PM, Zou X, Schulten K, Gaub HE: Effects of cytosine hydroxymethylation on DNA strand separation. Biophys J 2013, 104:208-215.

56. Seeman NC: DNA in a material world. Nature 2003, 421:427.

57. Van Mameren J, Vermeulen K, Wuite GJ, Peterman EJ: A polarized view on DNA under tension. J Chem Phys 2018, 148:123306

58. Backer AS, Biebricher AS, King GA, Wuite GJ, Heller I, Peterman EJ: Single-molecule polarization microscopy of DNA intercalators sheds light on the structure of S-DNA. Sci Adv 2019, 5:eaav1083.

59. Vologodskii A, Frank-Kamenetskii MD: DNA melting and energetics of the double helix. Phys Life Rev 2018, 25:1-21.

60. Stassi S, Marini M, Allione M, Lopatin S, Marson D, Laurini E,

$\bullet \quad$ Pricl S, Pirri CF, Ricciardi C, Di Fabrizio E: Nanomechanical DNA resonators for sensing and structural analysis of DNA-ligand complexes. Nat Commun 2019, 10:1-10
Developed a novel experimental technique to directly measure DNA elastic moduli.

61. Camunas-Soler J, Manosas M, Frutos S, Tulla-Puche J, Albericio F, Ritort F: Single-molecule kinetics and footprinting of DNA bis-intercalation: the paradigmatic case of Thiocoraline. Nucleic Acids Res 2015, 43:2767-2779.

62. Severin PM, Zou X, Gaub HE, Schulten K: Cytosine methylation alters DNA mechanical properties. Nucleic Acids Res 2011, 39:8740-8751

63. $\mathrm{Hu} \mathrm{Q}, \mathrm{Xu} \mathrm{S}$ : Sequence and chiral selectivity of drug-DNA interactions revealed by force spectroscopy. Angew Chem Int Ed 2014, 53:14135-14138.

64. Manosas M, Camunas-Soler J, Croquette V, Ritort F: Single molecule high-throughput footprinting of small and large DNA ligands. Nat Commun 2017, 8:1-12.

65. Kopperger E, List J, Madhira S, Rothfischer F, Lamb DC, Simmel FC: A self-assembled nanoscale robotic arm controlled by electric fields. Science 2018, 359:296-301.

66. Skoruppa E, Nomidis SK, Marko JF, Carlon E: Bend-induced twist waves and the structure of nucleosomal DNA. Phys Rev Lett 2018, 121:088101. 